\title{
=ALTERIDAD Realidad Aumentada y stellarium: astronomía para niños y niñas de cinco años
}

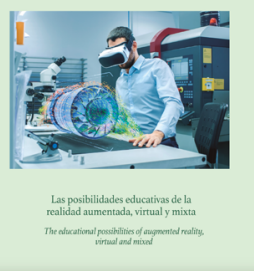

\author{
Augmented reality and stellarium: astronomy for \\ children offive years
}

iD Sandra Pérez-Lisboa es docente de la Universidad de Playa Ancha (Chile) (sandra.perez@upla.cl)

(https://orcid.org/0000-0002-0389-7242)

(iD Carmen Gloria Ríos-Binimelis es docente de la Universidad de Valparaíso (Chile) (carmengloria.rios@uv.cl) (https://orcid.org/0000-000 1-5800-6788)

(iD) Javier Castillo Allaria es docente de la Universidad de Playa Ancha (Chile) (javier.castillo@upla.cl) (https://orcid.org/0000-0001-8050-8231)

Recibido: 2019-10-17 / Revisado: 2019-12-02 / Aceptado: 2019-12-06 / Publicado: 2020-01-01

\section{Resumen}

Los educadores están llamados a favorecer la alfabetización digital, introduciendo las distintas herramientas tecnológicas con fines educativos ya que son parte del uso cotidiano de los niños y niñas. La Realidad Aumentada y el programa Stellarium, son poderosas herramientas para la enseñanza de la Astronomía, ya que permiten observar las estrellas, constelaciones y sistema solar, facilitando la explicación de los fenómenos celestes al educador/a. El presente estudio tuvo como objetivo analizar la intervención educativa con Realidad Aumentada y el programa Stellarium, en el desarrollo de lenguaje astronómico, específicamente el aspecto semántico y morfosintáctico sobre el sistema solar, estrellas y constelaciones, para niños y niñas de cinco años de edad. La intervención educativa fue llevada a cabo en el Aula Didáctica de la Universidad de Playa Ancha y aplicada a los niños y niñas del Nivel de Transición Mayor, de tres establecimientos municipales: Liceo San Felipe y las Escuelas Almendral y José Bernardo Suárez. Por medio de un estudio cuasi experimental (pre y post test), se analizó el avance que tuvieron los cincuenta y tres niños y niñas que participaron del estudio a través de dos test. Los resultados obtenidos evidenciaron el avance en el lenguaje científico al reconocer elementos del universo - aspecto semántico- expresar ideas y explicaciones sobre eventos astronómicos - aspecto morfosintáctico - que los niños y niñas conoció en la intervención educativa.

Descriptores: Lenguaje, astronomía, Realidad Aumentada, programa Stellarium.

\section{Abstract}

Educators are called to favor digital literacy, introducing the different technological tools for educational purposes since they are part of the daily use of children. Augmented reality and the Stellarium program are powerful tools for teaching astronomy, since they allow observing the stars, constellations and solar system, facilitating the explanation of celestial phenomena to the educator. The objective of this study was to analyze the educational intervention with augmented reality and the Stellarium program, in the development of astronomical language, specifically the semantic and morphosyntactic aspect of the solar system, stars and constellations, for five-year-old boys and girls. The educational intervention was carried out in the Didactic Classroom of the University of Playa Ancha and applied to the children of the Major Transition Level, of three municipal establishments: Liceo San Felipe and the Almendral and José Bernardo Suárez Schools. Through a quasi-experimental study (pre and post test), the progress of the fifty-three boys and girls who participated in the study through two tests was analyzed. The results obtained evidenced the progress in scientific language by recognizing elements of the universe — semantic aspect - expressing ideas and explanations about astronomical events - morphosyntactic aspect - that children knew in the educational intervention.

Keywords: Language, astronomy, augmented reality, Stellarium program.

Forma sugerida de citar: Pérez-Lisboa, S., Ríos-Binimelis, C. G., \& Castillo Allaria, J. (2020). Realidad Aumentada y stellarium: astronomía para niños y niñas de cinco años. Alteridad, 15(1), 25-35. https://doi.org/10.17163/alt.v15n1.2020.02 


\section{Introducción}

En el escenario educativo actual, las tecnologías de la comunicación y de la información, como la pizarra digital, Internet y sus recursos de aprendizajes (digital y virtual como software educativo), pueden constituirse en buenos aportes para la enseñanza de la Astronomía, ya que permiten a los niños y niñas observar las estrellas y constelaciones, tal como se verían durante la noche. De acuerdo a Vargas y Maya "la utilización de modelos simplificados para la enseñanza, constituye un recurso de extremada importancia para la enseñanza de la ciencia, porque al recurrir a experiencias visuales como cotidianas favorece a la formación de imágenes mentales de lo comprendido" (2007, p. 2). Los recursos educativos interactivos forman parte de esos medios tecnológicos que pueden favorecer un mejor aprendizaje, pues la utilización de materiales multimedia (simuladores y Realidad Aumentada), aportan opciones de enseñanza que facilitan un proceso educativo eficaz y de mejor calidad. Al respecto Bartolomé-Pina, García-Ruiz, y Aguaded señalan que "las tendencias no paran de crecer y a los nuevos dispositivos, cada vez más accesibles y ubicuos, se unen nuevas tendencias tecnológicas y estrategias didácticas como la Realidad Aumentada (2018, p. 34). Es importante señalar que de acuerdo a De Lima y Moreira (2019) que la incorporación de las TIC en la docencia contribuye a la potenciación de las acciones pedagógicas por parte de las educadoras.

La Astronomía es una de las ciencias más antiguas, pero dado el crecimiento tecnológico es también una ciencia moderna. La astronomía despierta la curiosidad en los niños y niñas, por ello es que el Ministerio de Educación, contempla dentro de las Bases Curriculares de la Educación Parvularia, en el Núcleo Exploración del Entorno Natural, la enseñanza de la astronomía. De esta forma, "se espera potenciar en las niñas y los niños, las habilidades, actitudes y conocimientos que les permitan comprender, apreciar y cuidar su entorno natural, poten- ciando su curiosidad y capacidad de asombro" (Subsecretaría de Educación Parvularia, 2017, p. 83). Desde pequeños los niños y niñas, poseen vivencias astronómicas concretas de observación del cielo en especial de la Luna, el Sol y las estrellas, las cuales, les provoca gran curiosidad, cuestionamientos y, sobre todo, una gran cantidad de preguntas e ideas diversas, que es indispensable rescatar a la hora de desarrollar la temática en las aulas. Al respecto Martin, Sexton, Franklin y Gerlovich (2005), señalan que las personas que han recibido educación en astronomía pueden comprender mejor el ambiente en el que viven y posicionarse de forma individual dentro del universo. Los niños y niñas forman sus percepciones de diferentes aspectos del macrocosmos, que contrastan con los nuevos conceptos científicos, en un intento por comprender los fenómenos del mundo natural (Vosniadou \& Brewer, 1992). Este proceso requiere una intervención temprana que proporcione ambientes y herramientas para el desarrollo de ideas y nuevos conceptos. Según Galperin (2015), para generar cambios en la forma de relacionar los fenómenos celestes con explicaciones "sobrenaturales", es necesario que el sistema educativo comience a incorporarlos dentro de su currículum a temprana edad.

El presente estudio tuvo como objetivo analizar la intervención educativa con Realidad Aumentada y el programa Stellarium, en el desarrollo de lenguaje astronómico, específicamente el aspecto semántico y morfosintáctico sobre el sistema solar, estrellas y constelaciones, para niños y niñas de cinco años de edad.

\subsection{Realidad Aumentada}

El término "Realidad Aumentada" (RA), fue usado por primera vez en 1992, por Tom Caudell para designar aquellas experiencias en las que se agrega información digital (por medio de texto, imagen, audio, vídeo, objetos 3D u otros) a una visión del mundo físico en tiempo real. Para realizar esta superposición de información virtual y real, se requiere la utilización de dispositivos tec- 
nológicos como: computadores con Webcams, o computadoras portátiles, tablets o smartphones. Para Gómez (2013), la RA es aquel entorno en el que tiene lugar, la integración de lo virtual y lo real. Por otro lado, Cabero y Barroso (2016), señala que la utilización de la RA en los contextos educativos permite mejorar las acciones formativas y por ende que los alumnos aumenten los niveles de aprendizaje mediante la creación de estos escenarios tecnológicos.

La aplicación de la RA en la educación se debe en gran medida, a la multiplicación en la utilización de dispositivos portátiles, smartphones y tablets y con un fin didáctico. Es lo que se conoce como mobile-learning, o m-learning, que Quinn (2000), lo señala como un tipo de e-learning a través de dispositivos móviles. Por su parte Basogain, Olabe, Espinosa, Rouèche y Olabe (2007) afirman que "la Realidad Aumentada no reemplaza el mundo real por uno virtual, sino al contrario, mantiene el mundo real que ve el usuario complementándolo con información virtual superpuesto al real" (2007, p. 1).

En el contexto educacional, la literatura señala que el uso de la RA influye positivamente en la motivación del alumnado. Uno de ellos es el de Cubillo, Gutiérrez, Gil, y Colmenar, mencionando que la RA "facilita, motiva y hace más agradable la explicación y asimilación de los contenidos tanto para los profesores como para los alumnos, estimula y motiva el aprendizaje" (2014, p. 248). Por otro lado, Reinoso dice que "...la RA refuerza el aprendizaje e incrementa la motivación por aprender” (2012, p. 371). Asimismo, Fonseca, Redondo y Valls (2016), dicen que la experiencia al utilizar imágenes del mundo real es satisfactorio y motivador porque permite una mayor comprensión del espacio. También esta herramienta según Prendes (2015), se usa para proporcionar prácticas a los estudiantes con algunas aplicaciones médicas. Ahora bien, es fundamental que los profesores estén capacitados para el uso de la aplicación, así podrán interactuar con los estudiantes en todo el proceso de aprendizaje (Oluwadare, 2015).

\subsection{Uso de simuladores en Astronomía}

La simulación según la Real Academia Española (RAE) viene del latín simulatio, -ōnis, y es la "acción y efecto de simular". Por consiguiente, consiste en situar al educando en un contexto que imite algún aspecto de la realidad y establecer en este ambiente, situaciones que él tendría que enfrentar en un contexto real. Salas (1995), señala que los educandos tienen mejor concentración en un objeto determinado al usar la simulación en la enseñanza.

El programa Stellarium tiene código abierto y es un software que posibilita al computador para utilizarlo como un planetario ya que se exhibe el cielo en 3D, tal cual se aprecia a simple vista con telescopio o binoculares. Señala las posiciones de las estrellas, el Sol, los planetas y la Luna, y dependiendo de la ubicación y la hora muestra cómo un observador ve el cielo. También simula fenómenos astronómicos como los eclipses lunares o solares, las lluvias de meteoritos y dibuja las constelaciones. Como herramienta educativa permite enseñar el cielo nocturno, ayuda la observación para astrónomos aficionados, o simplemente como curiosidad para incentivar el estudio de las ciencias planetarias.

Los diversos programas que se han creado por medio de la tecnología “...son importantes por la diversidad de posibilidades que nos ofrecen para crear nuevas escenografías comunicativas para la enseñanza" (Aguaded \& Cabero, 2014, p.71). Por ello que la utilización del programa Stellarium, que es un modelo simplificado de mucha importancia para la enseñanza de la ciencia, ya que al requerir a experiencias visuales como cotidianas permite la formación de imágenes mentales de lo comprendido, facilitando la aproximación de nociones de la astronomía (Vargas \& Maya, 2007). 


\subsection{La astronomía en la infancia}

La astronomía viene del lat. "astronomǐa, y este del gr. $\alpha \sigma \tau \rho o v o \mu i ́ \alpha$ astronomía”, se define como "Ciencia que trata de los astros, de su movimiento y de las leyes que lo rigen" (RAE, en línea). Como ciencia, estudia el universo más allá de la atmósfera de la Tierra, incluido el Sol, la Luna, los planetas y las estrellas (Brewer, 2001; Eliason \& Jenkins, 2011). Estos temas son de gran interés para los pequeños, el día y la noche, el Sol, la Luna y las estrellas, son parte de las experiencias que viven a diario porque están en su entorno. Para Educarchile "la astronomía en la escuela ofrece oportunidades valiosas para el fortalecimiento de la personalidad y el desarrollo de las habilidades cognitivas de los niños, que adquieren una visión más profunda del lugar que ocupan en el mundo y reciben herramientas" (en línea). Por ello que Ampartzaki y Kalogiannakis (2016), trabajan en un proyecto que tiene un enfoque multidisciplinario y basado en conceptos que consideran centrales en la enseñanza de la astronomía, los cuales son el aprendizaje espacial y pensamiento espacial.

Kallery (2010), señala que hay pocos estudios orientados a la astronomía y es relativamente limitado en comparación con el número de estudios sobre otras disciplinas. A nivel nacional en el informe 'Alcanzando las Estrellas: Hallazgos de las Cumbres Chileno-Estadounidenses de Educación y Difusión de la Astronomía' sólo se señala la enseñanza de la astronomía a nivel de educación Básica y Media, dejando de lado diversas propuestas que se están implementando.

La astronomía según Cabello (2011), nace de la curiosidad natural que tienen los infantes, para comprender los fenómenos naturales y así responder las preguntas con explicaciones más rigurosas. El desarrollar actitudes positivas hacia la astronomía, son una parte significativa de la alfabetización científica para las sociedades contemporáneas (Uçar \& Demircio lu, 2011). Además, según Nussbaum (1989), hay que tener en cuenta que los niños y niñas poseen expe- riencias astronómicas concretas e ideas y teorías ligadas a ellas, desde muy temprana edad. Por consiguiente se propone una intervención educativa para niños y niñas de cinco años de edad, en el aprendizaje de lenguaje de astronomía sobre el sistema solar, estrellas y constelaciones, utilizando la Realidad Aumentada y el programa Stellarium.

\subsection{Intervención educativa}

Las intervenciones educativas se aplicaron en el Aula Didáctica de la carrera de educación Parvularia de la Universidad de Playa Ancha, Campus San Felipe. En esta Aula Didáctica se "realiza actividades que trascienden el mero aprendizaje memorístico, pretenden capacitar para la comprensión de los contenidos a través de herramientas tecnológicas propias del S XXI" (Pérez-Lisboa \& Caldeiro, 2016, p. 184). Las herramientas utilizadas fueron el simulador Stellarium y RA con los cuales implementaron las experiencias educativas.

Los contenidos vistos por la educadora de párvulos en la intervención educativa fueron separados en tres unidades, divididas cada una de ellas, en cuatro sesiones de 60 minutos. Los contenidos vistos en cada unidad se detallan en la siguiente tabla.

El simulador Stellarium fue utilizado en cada sesión de 60 minutos por la educadora en la primera y tercera unidad. Se eligió esta herramienta ya que muestra el cielo nocturno y su evolución a lo largo del tiempo. En la primera unidad, esta herramienta permitió dar a conocer los niños y niñas las trayectorias que siguen los planetas en el cielo y los meses en los que pueden ser vistos. También señalar los movimientos de la luna y sus fases. En la tercera unidad el simulador se utilizó para enseñar que son las constelaciones, su ubicación y sus formas. Se seleccionó este programa debido a sus excelentes gráficas, ya que no solo se observan las líneas que unen las estrellas, sino que también muestra las imágenes de los seres mitológicos y objetos que éstas representan, permitiendo al mismo tiempo a la educadora contar leyendas 
asociadas a éstas, en las diferentes mitologías (clásica y de pueblos originarios).

La Realidad Aumentada fue usada en las unidades uno y dos ya que se dispuso de imágenes
3D de los planetas, satélites y estrellas, de manera que los menores pudieran apreciar sus principales características utilizando los distintos sentidos.

Tabla 1. Contenidos y sesiones

\begin{tabular}{|l|l|}
\hline \multirow{2}{*}{ Sistema Solar } & \multicolumn{1}{c|}{ Sesiones } \\
\hline \multirow{2}{*}{ Las } & El Sol \\
\cline { 2 - 2 } & Los planetas \\
\hline \multirow{2}{*}{ Lastrellas } & Cometas \\
\hline & La Luna \\
\hline \multirow{2}{*}{ Constelaciones } & Qué son las estrellas \\
\hline & Evolución estelar \\
\hline & Diagrama HR \\
\hline & Agrupaciones estelares \\
\hline & Que son las constelaciones \\
\hline & Historias y leyendas del cielo \\
\hline & Constelaciones de los pueblos originarios \\
\hline & Ubicando las constelaciones \\
\hline
\end{tabular}

\section{Metodología}

El diseño de la presente investigación, corresponde a un estudio de tipo cuasi-experimental con pre-test/post-test (Hernández Sampieri, Fernández Collado \& Baptista, 2014) debido a que los investigadores solo se limitan a observar y analizar el proceso de enseñanza-aprendizaje que se lleva a cabo mediado por las TIC.

Los test que se les aplicaron a la población objeto del estudio, fueron dos, el primero analizó el nivel que tenían los niños y niñas del aspecto semántico y el segundo analizó el nivel que tenían los párvulos del aspecto morfosintáctico, del lenguaje astronómico enseñado en la intervención educativa. Los test fueron elaborados por lo/as investigadores y validados por juicio de expertos. El primer test está compuesto por ocho láminas, cada lámina tiene cuatro objetos astronómicos, donde el niño o niña debe señalar el objeto nombrado por el examinador. El segundo test tiene catorce preguntas, en las primeras tres deben completar las oraciones, en las tres siguientes deben identificar el término incorrecto, en las tres sucesivas deben señalar lo que ocurre con cada cuerpo celeste, en las tres que continúan deben realizar una oración con el elemento que se les menciona y en las dos últimas deben decir todas las cosas que saben del cuerpo celeste nombrado. Para ambos test se le da un punto si la respuesta esta errónea y dos puntos si la respuesta esta correcta.

La muestra del estudio correspondió a cincuenta y tres niños y niñas de los establecimientos municipales: Liceo San Felipe (dieseis), Escuela Almendral (dieciocho) y Escuela José Bernardo Suárez (diecinueve). La intervención educativa fue llevada a cabo en 12 sesiones, las cuales fueron desarrolladas en el Aula Didáctica de la Universidad de Playa Ancha, Campus San Felipe. El grupo de trabajo fue conformado por el investigador principal, una educadora de párvulos, un asesor científico y un ingeniero en informática. 


\section{Resultados y discusión}

La presente investigación cuasi experimental, analiza los resultados de una intervención educativa utilizando la Realidad Aumentada y el programa Stellarium, para niños y niñas de cinco años de edad en el aprendizaje de la astronomía. Los cincuenta y tres párvulos que participaron de la inter-

\section{Liceo San Felipe}

Figura 1. Resultados evaluación aspecto semántico

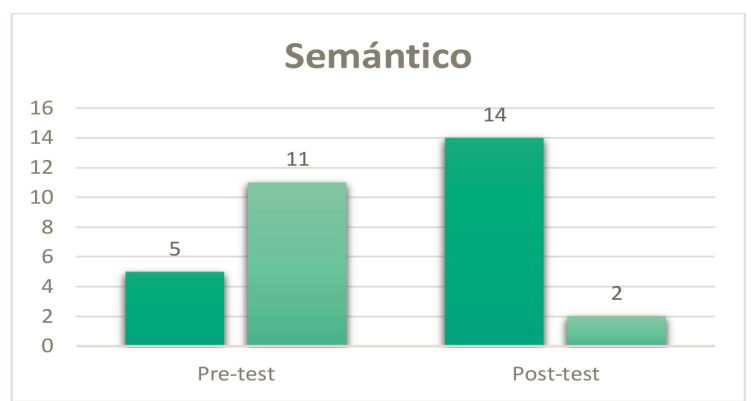

\section{Escuela Almendral}

Figura 2. Resultados evaluación aspecto semántico

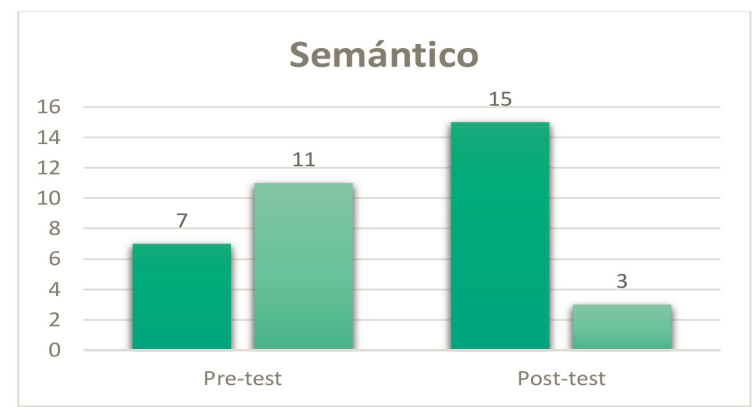

\section{Escuela José Bernardo Suárez}

Figura 3. Resultados evaluación aspecto semántico

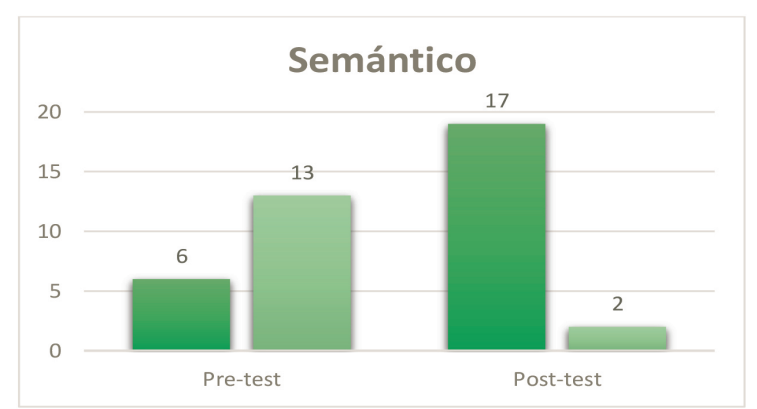

vención educativa fueron desarrollando los aspectos semánticos y morfosintácticos de los aprendizajes sobre el sistema solar, estrellas y constelaciones.

La aplicación del test que evalúo el aspecto semántico, dio a conocer el avance en el vocabulario de los conceptos de sol, luna, planeta Tierra, planeta Marte, planeta Saturno, constelación, estrella y cometa. A continuación, se examinarán los resultados obtenidos del pre y pos test.

Los dieciséis niños y niñas que participaron de la intervención del Liceo San Felipe, Figura 1, da cuenta que la mayoría fueron aprendiendo los conceptos. En el pre-test sólo cinco conocían los términos, en el post-test catorce niños y niñas lograron identificar los conceptos enseñados.

De la Escuela Almendral, los dieciocho niños y niñas que participaron, Figura 2, se observa que casi el total de ellos fueron asimilando los conceptos evaluados. En el pre-test había sólo seis identificaban los términos, en el post-test diecisiete párvulos conocían todos los conceptos vistos en la intervención educativa.

Los diecinueve niños y niñas que participaron de la intervención de la escuela José Bernardo Suárez, Figura 3, se observa que la mayoría fueron aprendiendo los conceptos. En el pre-test sólo seis conocían los términos, en el post-test diecisiete párvulos lograron identificar los conceptos enseñados. 
El análisis de los tres establecimientos da cuenta un avance significativo en el aprendizaje de los conceptos, el aspecto semántico "se relaciona con la toma de conciencia y la organización de los contenidos lingüísticos en la memoria y con la fluidez para establecer relaciones significativas que tenga la persona" (Pérez- Lisboa, 2017, p. 6). $\mathrm{Al}$ respecto Kallery (2010), señala que los conceptos astronómicos como el cambio de día y noche, la esfericidad de la tierra que se consideran difíciles para niños pequeños, pueden ser asequibles si se les presentan con estrategias de enseñanza que los motivan y despierten su interés. Según Pérez, Castillo y Ríos (2017), "la RA permitió experiencias de aprendizajes cercanas y significativas de los niños y niñas", permitiendo el aprendizaje. Por otro lado, Longhini y Menezes (2010), señalan que el uso del programa Stellarium es una herramienta que revela un alto potencial educativo, ya que tiene más de 600000 estrellas; ilustraciones de las constelaciones; planetas del sistema solar y sus satélites, eclipses solares y lunares, entre otros. Así también el Stellarium refuerza significativamente los contenidos que se ven en la clase (Sá Muniz \& da Silva, 2015).

Continuando con el análisis, en el aspecto morfosintáctico se evalúo completar frases, detectar errores en frases, establecimientos de relaciones causales, construcción de frases y enumeración de elementos pertenecientes a determinadas categorías verbales. A continuación, se dan a conocer los resultados de cada establecimiento.

\section{Liceo San Felipe}

Figura 4. Resultados evaluación aspecto morfosintáctico

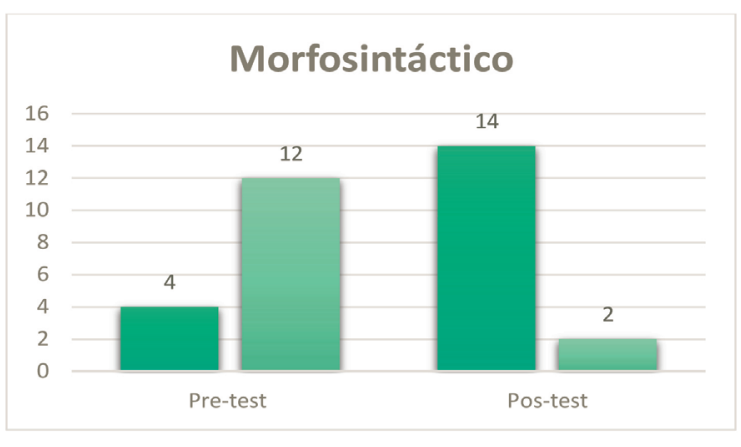

\section{Escuela Almendral}

Figura 5. Resultados evaluación aspecto morfosintáctico

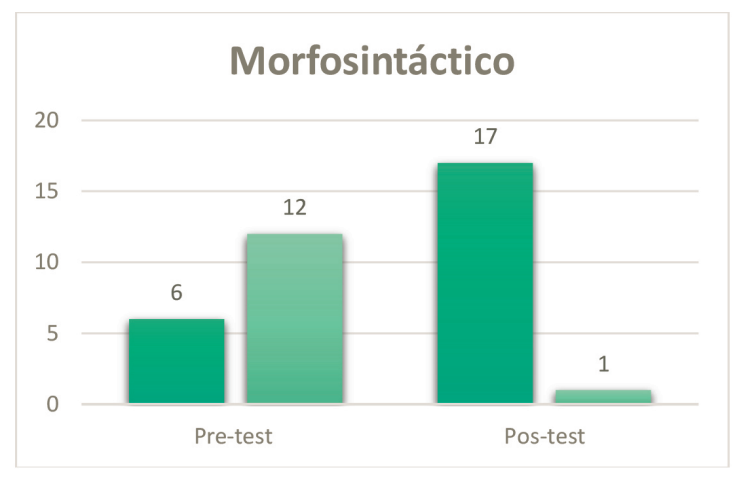

Del Liceo San Felipe, la Figura 4, da a conocer que los niños y niñas avanzaron significativamente en la construcción de oraciones, al utilizar el lenguaje astronómico que fueron aprendiendo. Al finalizar la intervención educativa, el resultado del post-test señala que, catorce párvulos pudieron avanzar en la estructura gramatical al usar clases de palabras y componerlas en el orden de los elementos oracionales, lo que realizan sólo cuatro de ellos según el pre-test.

Los dieciocho niños y niñas que participaron en la intervención educativa de la Escuela Almendral, Figura 5, avanzaron en la composición de oraciones ya que en el pre-test sólo seis lo realizaban. Al finalizar la intervención educativa, diecisiete párvulos fueron usando palabras y artificios para la composición y el orden de los elementos oracionales al explicar los fenómenos astronómicos. 


\section{Escuela José Bernardo Suárez}

Figura 6. Resultados evaluación aspecto morfosintácticomorfosintáctico

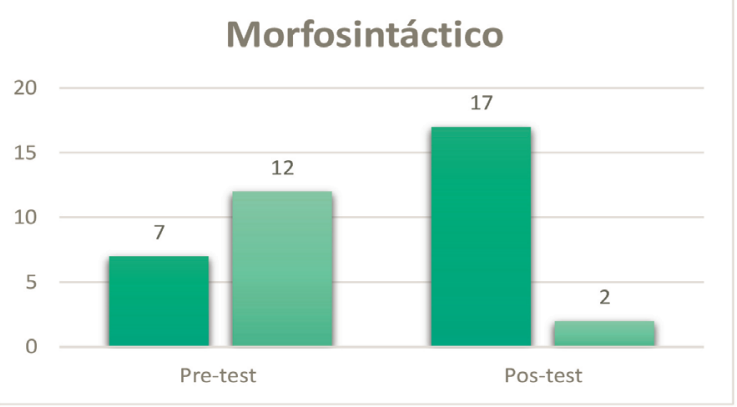

De los diecinueve niños y niñas que participaron del estudio, Figura 6, en el pre-test siete estructuraban adecuadamente una oración. Al término de la intervención diecisiete usaban clases de palabras y las componían en orden de los elementos oracionales, cuando explicaban los conceptos astronómicos enseñados.

El aspecto morfosintáctico involucra entender las diferentes partes de la oración y la función que tiene cada una en el texto total (Díaz \& Álvarez-Salamanca, 2006). Según Peterson y French (2008), los niños y niñas aprenden los componentes lingüísticos de las explicaciones de la mezcla de colores, al participar en las discusiones con las educadoras y compañeros. En este contexto el programa Stellarium al simular los movimientos de la Tierra y las fases de la Luna permite a los estudiantes explicar lo que están observando (Andrade, Silva \& Araújo, 2009). Asimismo, Reinoso (2012) señala que la RA con la visualización de objetos en $3 \mathrm{D}$ lo/as estudiantes pueden explorar y dar a conocer las propiedades físicas de estos. Por consiguiente, ambas herramientas, les permitieron experiencias visuales para avanzar en la construcción de lenguaje científico al comunicar fenómenos observados.

\section{Conclusiones}

$\mathrm{Al}$ ser la Astronomía una ciencia que requiere de la observación nocturna, los simuladores como el programa Stellarium y la Realidad Aumentada, pueden suplir este inconveniente mostrando de una forma realista el cielo nocturno, lo que permite a los niños y niñas observar las estrellas, constelaciones y sistema solar tal como las vería en la noche, facilitando la explicación de los fenómenos celestes al educador.

Es por esto, que las educadoras de párvulos en el presente, deben diversificar las oportunidades incorporando medios innovadores en el proceso de enseñanza y aprendizaje. Utilizar recursos tecnológicos en la educación, es una contribución metodológica y representa un desafío en modificar el repertorio de las prácticas pedagógicas para promover en las educadoras su creatividad.

A partir de los resultados obtenidos en la intervención educativa se observa que:

- El uso de la Realidad Aumentada y simuladores al servicio de la educación, ha permitido diversificar la enseñanza para mejorar el aprendizaje. La niña y el niño es el protagonista y forjador de su propio conocimiento, por medio de sus habilidades y destrezas en el manejo de los recursos tecnológicos que tiene a su disposición.

- Los resultados de los test aplicados en los tres colegios muestran que la incorporación de las TIC en la intervención educativa, se convierten en un apoyo en el aprendizaje de los conceptos astronómicos y también, del desarrollo del lenguaje científico, por parte de los niños y niñas, lo que concuerda con el estudio de Pérez-Lisboa (2017).

- Se propuso el desarrollo de esta intervención educativa, como una herramienta de apoyo para el estudio de las estrellas y constelaciones. En esta intervención educativa, se observó el gran interés que despiertan los recursos multimedia en los niños, la gran motivación con que hacen uso de ellos y los enormes alcances conceptuales, reflejados en los desempeños obtenidos y en el dominio y propiedad sobre los cono- 
cimientos adquiridos, lo que concuerda con los estudios de Cubillo et al. (2014).

- Los contenidos trabajados con este material y la forma como se presentan en esta intervención educativa, mediante imágenes 3D y simulaciones, generaron en los estudiantes gran curiosidad e interés por conocer cada uno de los temas, permitiéndoles, además, mejorar su desempeño, ya que están concentrados en las actividades que realizan, lo que concuerda con los estudios Giasiranis y Sofos (2016).

- Se resalta con esta investigación la importancia que tiene este tipo de recursos didácticos e interactivos para la enseñanza-aprendizaje. La Astronomía, al ser una ciencia que se basa en la observación y especialmente de avistamiento nocturno se ve favorecida con la incorporación de estas tecnologías para su divulgación y enseñanza.

- Las TIC, son un medio didáctico recreativo donde el profesor y el estudiante no solo interactúan con objetos inanimados, si no que se les puede generar movimiento, lo que es muy divertido para aprender y enseñar de una manera didáctica. De esta forma las TIC se convierten en una herramienta que permite realizar representaciones de forma de conocer las características y atributos del universo, como lo señala en MINEDUC (2001).

- En relación a las limitaciones que tuvo el presente estudio fue el poco tiempo de implementación, el que duró sólo un semestre y no dos como habían sido las intervenciones de los años anteriores, esto se debió al poco financiamiento que se le dio al proyecto, que no permitió seguir pagando a la educadora de párvulos que estuvo a cargo.

Como proyecciones el equipo investigador pretende seguir buscando alternativas de financiamiento para continuar en la enseñanza de la astronomía en la Educación Parvularia.

\section{Apoyos y agradecimientos}

Este trabajo fue respaldado por la Dirección General de Investigación de la Universidad de Playa Ancha

\section{Consideraciones éticas}

Antes de evaluar a los niños y niñas que participaron de la intervención educativa, los padres, madres y apoderados dieron su consentimiento informado, además aceptaron que se les sacaran fotografía y los gravaran para que estas fueran ocupadas en este artículo. Asimismo, las educadoras de párvulos que implementaron el proyecto dieron también su consentimiento informado para este artículo.

\section{Expresión de gratitud}

Queremos agradecer a todos los niños y niñas que participaron de la investigación, el interés por aprender, la alegría, el asombro, el cariño, el respeto y la curiosidad que demostraron, nos permitió evidenciar que lo que estabas haciendo era muy importante para ellos. A sus padres, madres y familiares por autorizar la participación en el estudio. A las directoras, jefas de la unidad técnica pedagógicas, educadoras de párvulos por creer en nuestro proyecto. A las educadoras de párvulos que implementaron la intervención educativa. Por último, agradecer a la Dirección General de Investigación y la Facultad de Ingeniería de la Universidad de Playa Ancha quienes nos ayudaron a financiar esta investigación.

\section{Referencias bibliográficas}

Aguaded, I., \& Cabero, J. (2014). Avances y retos en la promoción de la innovación didáctica con las tecnologías emergentes e interactivas. Educar especial 30 aniversario 67-83.

https://doi.org/10.5565/rev/educar.691

Ampartzaki, M., \& Kalogiannakis, M. (2016). Astronomy in Early Childhood Education: 
A Concept-Based Approach. Early Childhood Education Journal, 44(2), 169-179.

Andrade, M., Silva, J., \& Araújo, A. (2009). utilização do software stellarium Para o ensino de astronomia. (http://bit.ly/2DX1Mwn).

Bartolomé-Pina, A., García-Ruiz, R., \& Aguaded, I. (2018). Blended learning: panorama y perspectivas. Revista Iberoamericana de Educación a Distancia, 21(1), 33-56. http://dx.doi.org/10.5944/ried.21.1.18842

Basogain, X., Olabe, M., Espinosa, K., Rouèche, C., \& Olabe, J. (2007). Realidad Aumentada en la Educación: una tecnología emergente. (http://bit.ly/36h35Co).

Brewer, J. A. (2001). Introduction to early childhood education: Preschool through primary grades. USA: By Allyn and Bacon.

Cabello, M.J. (2011). Ciencia en educación infantil: la importancia de un "rincón de observación y

experimentación" o "de los experimentos" en nuestras aulas. Revista, 10.

Cabero, J., \& Barroso, J.M. (2016). Posibilidades educativas de la Realidad Aumentada. Journal of New Approaches in Educational Reasearch, 5(1), 46-52

Cubillo, J., Gutiérrez, S., Gil, M., \& Colmenar, A. (2014). Recursos digitales autónomos mediante Realidad Aumentada. RIED. Revista Iberoamericana de Educación a Distancia, 17(2), 241-274.

De Lima, M. R., \& Moreira de Andrade, I. (2019). Significaciones docentes sobre la integración de tecnologías digitales en prácticas pedagógicas. Alteridad, 14(1), 12-25. https://doi.org/10.17163/alt.v14n1.2019.01

Díaz, C., \& Álvarez-Salamanca, E. (2006). La importancia de potenciar tempranamente las competencias lingüísticas en la etapa inicial. Boletín de Investigación Educacional, 21(1), 115-137. Facultad de Educación. Pontificia Universidad Católica de Chile. Santiago.

Educarchile. (http://bit.ly/2S26Bwq).

Eliason, C. F., \& Jenkins, L.T. (2011). A practical guide to early childhood curriculum's. Pearson.

Fonseca, D., Redondo.E., \& Valls, F. (2016). Motivation and Academic Improvement Using Augmented Reality for 3D Architectural Visualization. Education in the Knowledge Society, 17(1), 45- 64.
Galperin, D. (2015). Propuestas didácticas para la enseñanza de la Astronomía. (http://bit.ly/34Gfy14).

Giasiranis, S., \& Sofos, L. (2016). Production and Evaluation of Educational Material Using Augmented Reality for Teaching the Module of "Representation of the Information on Computers" in Junior High School. Creative Education, 07(09), 1270-1291. (http://bit.ly/2s7YWlq).

Gómez, M. (2013). Educación Aumentada con Realidad Aumentada. En 3er Congreso Internacional sobre Buenas Prácticas con TIC en la Investigación y la Docencia. Universidad de Málaga. 23-25 de octubre.

Hernández, R., Fernández, C., \& Baptista, P. (2008). Metodología de la Investigación. México: McGraw-Hill.

Kallery, M. (2010). Astronomical concepts and events awareness for young children. International Journal of Science Education, 33(3), 341-369. http://dx.doi.org/10.1080/09500690903469082

Longhini, M., \& Menezes, D. (2010). Objeto virtual de aprendizagem no ensino de astronomia: algumas situaçõesproblema propostas a partir do Software stellarium. Caderno Brasileiro de Ensino de Física, 27(3), 433-448. https://doi.org/10.5007/2175-7941.2010v27n3p433

Martin, R., Sexton, C., Franklin, T., \& Gerlovich, J. (2005). Teaching science for all children an inquiry approach. Boston: Pearson Publishing.

MINEDUC (2001). La Educación Parvularia en Chile. Unidad de Educación Parvularia División de Educación General Ministerio de Educación República de Chile. (http://bit.ly/34z2N8P).

Nussbaum, J. (1989). La tierra como cuerpo cósmico. En R. Driver, E., Guesne, y A. Tiberghien, Ideas científicas en la infancia y la adolescencia. Madrid: Morata.

Oluwadare, F. (2015). ICT Use in Preschool Science Education: A Case Study of Some Private Nursery Schools in Ekiti State. Journal of Education and Practice, 6(31), 75- 79.

Pérez-Lisboa, S. (2017). Descubriendo el lenguaje a través de la Realidad Aumentada y la pizarra digital. Revista Electrónica Educare, 21(3), 1-13. http://dx.doi.org/10.15359/ree.21-3.14 
Pérez-Lisboa, S., \& Caldeiro, M. (2016). Aprendiendo Ciencia en el Aula didáctica: retos y potencialidades. Ecos de la Academia, 2, 182-185.

Pérez, S., Castillo, J., \& Ríos, C. G. (2017). Realidad Aumentada y pizarra digital interactiva en la construcción de habilidades científicas. Una propuesta metodológica para niños y niñas del nivel transición mayor en el aprendizaje de la física. En: Investigaciones y experiencias en educación virtual. Bogotá, Colombia: Ediciones UGC.

Prendes, C. (2015). Realidad Aumentada y educación: análisis de experiencias prácticas. Revista de Medios y Educación, 46, 187 -203. http://dx.doi.org/10.12795/pixelbit.2015.i46.12

Peterson, S., \& French, L. (2008). Supporting young children's explanations through inquiry science in preschool. Early Childhood Research Quarterly, 23, 395-408.

RAE. Real Academia Española. (https://dle.rae.es/?id=XvyuZ0x).

Salas, R. (1995). La simulación como método de enseñanza y aprendizaje. Educación Médica Superior, 9(1).
Sá Muniz, T., \& da Silva, E. (2015). Método didático para o ensino de Astronomia: utilização do software Stellarium em conjunto com aulas expositivas no ensino médio. Revista Eletrônica da Fainor, Vitória da Conquista, 8(2), 87-97.

Subsecretaría de Educación Parvularia. Bases Curriculares de Educación Parvularia. Santiago, Chile: MINEDUC.

Quinn, C. (2000). mLearning. Mobile, Wireless, In-Your-Pocket Learning. Linezine. Fall 2000. (http://bit.ly/343PT27).

Vargas, J., \& Maya, O. (2007). Enseñanza de la astronomía con material interactivo. (http://bit.ly/2Yw06Dr).

Vosniadou, S., \& Brewer, W. (1992). Mental models of the earth: A study of conceptual change in childhood. Cognitive Psychology, 24(4), 535585. (http://bit.ly/34vDvIn)

Uçar, S., \& Demircioğlu, T. (2011). Changes in preservice teacher attitudes toward astronomy within a semester-long astronomy instruction and fouryear-long teacher training programme. Journal Science Education Technology, 20(1), 65-73. https://doi.org/10.1007/s10956-010-9234-7 